\title{
Lettre à la rédaction :
}

\section{Une supplémentation orale en créatine} interfère t'elle sur la créatinine plasmatique?

\author{
Letter to the editor: \\ Does alimentary supplement with creatine \\ interfere on plasmatic creatinine values?
}

\section{Tiphaine GAILLARD, Loïc EMILE, Renaud RIVIERES, Corinne CIVADIER}

Service de Biochimie, Pharmacologie et Toxicologie Cliniques, Hôpital d'Instruction des Armées Sainte Anne BP 600 - 83800 TOULON ARMÉES - Tél : 0494099206

(Reçu le 27 octobre 2003 ; accepté le 10 décembre 2003)

L'hospitalisation d'un patient consommateur de créatine nous a amené à suspecter une interférence sur l'évaluation de la créatinine plasmatique.

La créatine est une petite molécule dérivée d'un acide aminé ; elle provient en partie de l'alimentation (viande rouge) ou est synthétisée par l'organisme au niveau du foie et libérée dans le flux sanguin. Le taux de créatine sérique est voisin de $15 \mathrm{mg} / \mathrm{l}$ soit $115 \mu \mathrm{mol} / \mathrm{l}$. Une partie de la créatine véhiculée par le sang est transformée dans le muscle par une créatine kinase en phosphocréatine, molécule à potentiel énergétique élevé qui intervient lors des contractions musculaires.

Une supplémentation alimentaire en créatine vise essentiellement à permettre une augmentation de la masse musculaire $(1,2)$. La créatine utilisée en tant que supplément nutritionnel est parfois associée à d'autres substances dopantes ; elle joue alors un rôle d'excipient. Elle est généralement consommée à raison de 20 grammes par 24 heures, en 4 à 6 prises. Seule une faible quantité de la créatine ingérée (5 à $25 \%$ ) serait effectivement absorbée ; elle est rapidement éliminée dans les selles et les urines, l'élimination totale intervenant dans les 24 heures suivant la prise (3). Certains sujets utilisent la créatine par injections intra- veineuses ; il faut signaler les conséquences extrêmement lourdes pouvant résulter d'une telle pratique : des troubles cardiovasculaires et des néphropathies graves peuvent survenir.

Nous avons entrepris l'étude de l'interférence de la créatine sur le dosage de la créatinine plasmatique. Dans la mesure où les risques d'effets secondaires pathogènes d'une supplémentation alimentaire en créatine sont limités, nous avons décidé de reproduire chez deux sujets sains de sexe différent la prise orale d'une dose unique de $5 \mathrm{~g}$ de monohydrate de créatine pur (Anastore ${ }^{\circledR}$ ) dans $100 \mathrm{ml}$ d'eau (soit $383 \mathrm{mmol} / \mathrm{l}$ ).

Des dosages de la créatinine sur plasma hépariné sont effectués pour chacun des sujets à différents temps : le premier avant l'ingestion de la créatine, correspondant au taux de créatinine propre de chaque patient $\left(\mathrm{T}_{0}\right)$, les suivants à $T_{0,5 h}, T_{1 h}, T_{1,5 h}, T_{2,5 h}, T_{3,5 h}, T_{5,5 h}$.

Deux méthodes de détermination de la créatinine ont été appliquées, conformément aux recommandations des fabricants ; la première est colorimétrique (4), utilise la réaction de Jaffé et est adaptée sur $\mathrm{RxL}$ Dimension ${ }^{\circledR}$ (Dade Behring) ; la seconde est enzymatique, fait appel à une créatine iminohydrolase et est adaptée sur Vitros@ (Ortho-clinical Diagnostic) $(4,5)$. 
La créatine plasmatique a été mesurée, après transformation en créatinine par hydrolyse acide à chaud, par une méthode colorimétrique basée sur la réaction de Jaffé.

Les variations de la créatininémie sont représentées sur la figure 1 .

Des dilutions ont été nécessaires pour évaluer la teneur en créatinine de certains échantillons analysés par la méthode enzymatique, cette dernière entraînant une grande interférence entre les deux substances (code erreur $\mathrm{OR}, \mathrm{DP}$ et $\mathrm{PF}$ correspondant à des résultats en dehors de l'intervalle de linéarité et à une déplétion de substrat durant le processus analytique).

Le pic de créatine ( $>500 \mu \mathrm{mol} / \mathrm{l}$ ) survient une heure après la prise orale. Il correspond à celui de créatinine mesuré par la méthode enzymatique. Le retour aux valeurs normales se produit pour chaque patient en 3 à 5 heures.

Nous avons ainsi pu observer :

- que la créatine ingérée dans un but d'amélioration des performances physiques n'est que peu absorbée dans l'organisme ;

- que les techniques enzymatiques utilisant la créatine iminohydrolase ne permettent pas de mesurer de façon fiable la concentration en créatinine en présence de créatine, contrairement aux méthodes colorimétriques dérivées de la réaction de Jaffé.

\section{Remerciements}

les auteurs remercient Michel Lhermitte et Bernard Cartigny pour les informations apportées lors de l'élaboration de cette étude.

\section{Références}

1. Poortmans J.R., Francaux M. Adverse effects of creatine supplementation : fact or fiction? Sports Med. 2000 ; $30: 155-70$.

2. Engelhardt M., Neumann G., Berbalk A., Reuter I. Creatine supplementation in endurance sports. Med. Sci. Sports Exerc. $1998 ; 30: 1123-9$.

3. Cartigny B., Azaroual N., Mille-Hamard L, Imbenotte M., Kintz P., Vermeersch G., Lhermitte M. 1H-NMR urine analysis as an effective tool to detect creatine supplementation. J Anal. Toxicol., $2002 ; 26: 355-9$.

4. Murray R.L. Creatine. In : Pesce A.J., Kaplan L.A. Methods in clinical chemistry. St Louis : Mosby, 1987 : 876-81.

5. Sena S.F., Syed D., McComb R.B.. Effect of high creatine content on the Kodak single-slide method for creatinine. Clin. Chem. 1988 ; 34 : 594-5.

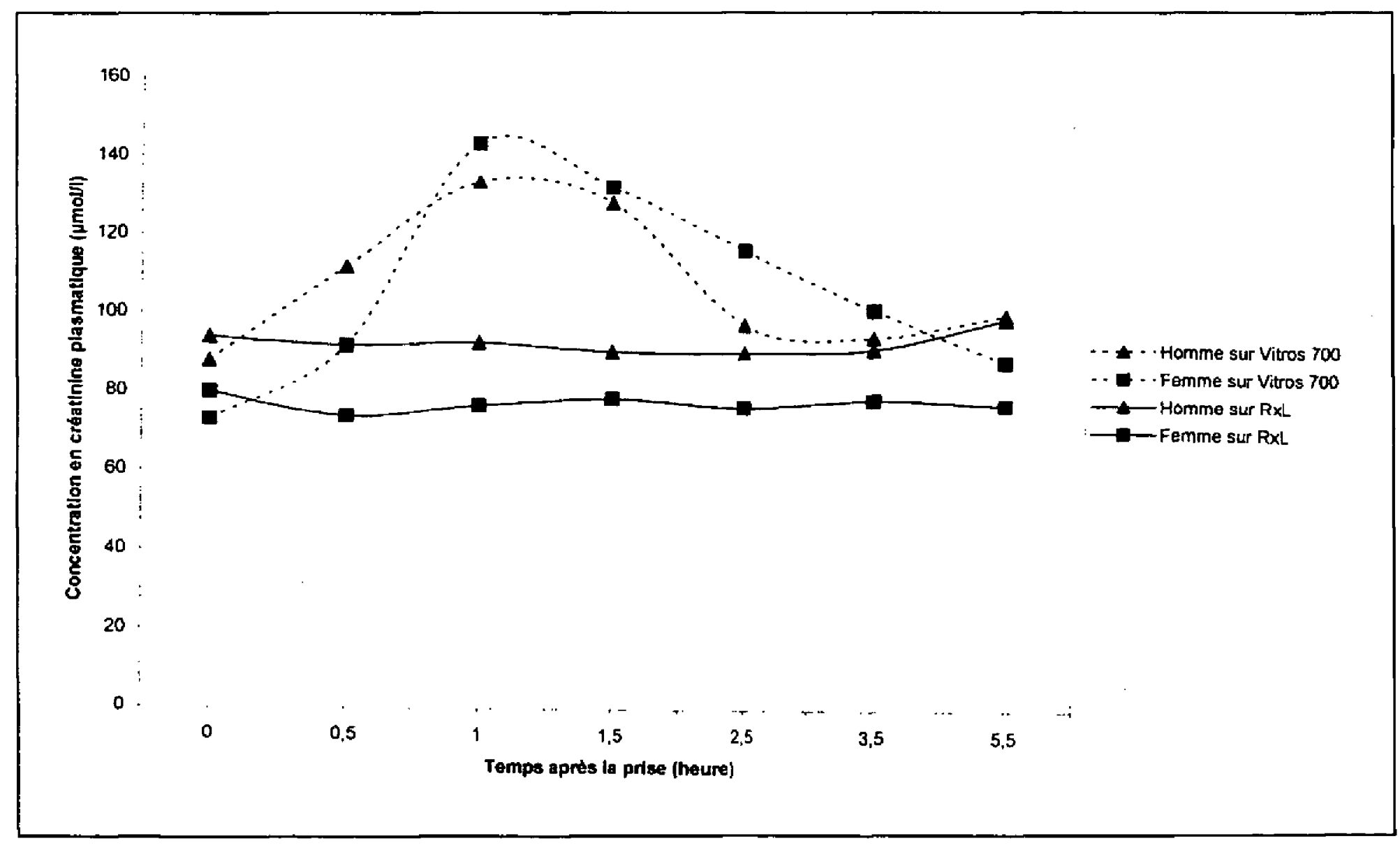

Figure I : Évolution de la concentration de la créatinine plasmatique après prise orale de $5 \mathrm{~g}$ de créatine : mesures par deux méthodes différentes. 